Ann. Génét. Sél. anim., I977, 9 (I) I-I3.

\title{
Conduite d'une population témoin de Lapins Evolution à court terme du coefficient de consanguinité selon le schéma d'accouplement
}

\author{
G. MATHERON et C. CHEVALET \\ Station d'Amélioration Génétique des Animaux, \\ Labovatoire de Génétique cellulaive, \\ Centre de Recherches de Toulouse, I.N.R.A., \\ 3I320 Castanct Tolosan BP I2
}

\begin{abstract}
Résumé
Différents modes de conduites d'une population témoin de lapins sont comparés vis-à-vis de l'évolution prévisible de la consanguinité. Deux types de schémas d'accouplement reposant sur une rotation entre onze familles sont discutés par référence aux accouplements au hasard admettant les mêmes règles de renouvellement. Sur le plan théorique on définit de façon rigoureuse une taille efficace de consanguinité pour le cas où les distributions du nombre de descendants par parent dépendent du sexe des descendants. Sur le plan pratique, l'accent est mis sur le comportement des premières générations et sur l'influence des parentés initiales entre fondateurs, qui ne sont pas pris en compte dans la notion de taille efficace. Les schémas sont comparés sur les dix premières générations en construisant les généalogies et en calculant les coefficients de consanguinité individuels : les comparaisons portent sur les valeurs moyennes et sur la variabilité des coefficients de consanguinité. On montre l'intérêt d'un schéma fondé sur des considérations théoriques : compte tenu des parentés entre animaux fondateurs, il permet de réduire durablement la consanguinité moyenne, de 3 p. roo par rapport aux croisements au hasard, et au bout de quelques générations il uniformise les coefficients individuels, évitant l'apparition fortuite d'animaux très consanguins.
\end{abstract}

\section{Introduction}

Depuis I970, la Station d'Amélioration Génétique des animaux INRA TOULOU$\mathrm{SE}$ a mis en place un programme de recherche appliquée à l'amélioration génétique du lapin pour la production de viande. Dans ce cadre, nous avons entrepris la sélection de souches (troupeaux fermés d'effectifs limités) sur la productivité numérique au sevrage; il paraissait utile de prévoir, simultanément, la mesure de l'évolution génétique des populations sélectionnées. Cette préoccupation est commune aux diverses espèces animales et a fait l'objet d'une mise au point récente (bull. Tech. Dép. Génét. anim., I9, r975). Nous avons étudié la constitution et le mode de conduite d'un troupeau de référence devant servir de témoin par 
rapport aux souches que nous sélectionnons. Ce troupeau est fermé et d'effectif limité.

La mesure de l'évolution génétique des troupeaux se fait en mesurant pour chaque caractère et intra génération les écarts, ou les moyennes d'un troupeau témoin, et en étudiant la régression de ces écarts sur le numéro de génération. La méthode suppose notamment que la moyenne génotypique du troupeau témoin reste constante au cours des générations successives et du temps. L'objectif est donc de minimiser la dérive génétique, inévitable dans une population d'effectif limité, et de veiller à ce que la moyenne génotypique du troupeau témoin n'évolue pas malgré tout, en l'absence de sélection artificielle, du fait de la sélection naturelle ou du mode de conduite.

Plusieurs auteurs ont étudié sur le plan théorique l'évolution de la consanguinité dans de tels troupeaux, compte tenu de l'effectif de reproducteurs mâles et femelles et du plan d'accouplement. Les schémas d'accouplement en effectif limité qui minimisent la dérive génétique ont été étudiés en détail par WRIGHT (I92I) et plus récemment par Kimura et CROW (I963), WRIGHT (I969) et CoCKERHAM (I970). Si l'on n'envisage pas une partition de la population en lignées évoluant indépendamment, les schémas les plus favorables parmi ceux pour lesquels le taux asymptotique de disparition des hétérozygotes a été calculé sont ceux définis par Kimura et $C_{\text {ROW }}$ : accouplement rotatif entre demi frères et demi sœurs, accouplements circulaires entre couples de reproducteurs, accouplement circulaire entre sous populations. Pour tous ces croisements la taille efficace $\mathrm{Ng}$ est proportionnelle au carré du nombre de paires ou de groupes d'individus accouplés. Ces schémas sont intéressants vis-à-vis de l'évolution asymptotique et à ce point de vue meilleurs que ceux de WRIGHT qui visent à éviter les accouplements entre individus apparentés jusqu'à un degré fixé. Ils ont cependant l'inconvénient de produire dès les premières générations des individus assez fortement consanguins, ce qui peut être préjudiciable à leur survie. CoC KERHAM (I970) a proposé un schéma alliant les propriétés de ceux de CROW et KIMURA et de WRIGHT, en envisageant des accouplements circulaires avec un décalage à chaque génération de la distance entre individus accouplés.

Ces comparaisons entre schémas portent sur la taille efficace $\mathrm{Ng}$ de la population, c'est-à-dire sur la vitesse asymptotique de disparition des hétérozygotes. Ce critère n'est pas intéressant si l'on veut étudier l'évolution à court ou à moyen terme d'une population, il est particulièrement mal adapté pour étudier à court terme des schémas d'accouplements circulaires. La comparaison du schéma de WRIGHT (I92I) avec 32 individus $(\mathrm{Ng} \simeq 6 \mathrm{I})$ et du schéma circulaire de KIMURA et CRow (I963) avec I6 individus $(\mathrm{Ng} \simeq 66)$ est significative à cet égard. Les tailles efficaces sont comparables, avec un léger avantage pour le schéma circulaire, mais le schéma de WRIGHT est de loin le plus intéressant : après ro générations la consanguinité est dix fois plus faible $(0,047$ contre 0,432$)$, après 50 générations elle est encore deux fois plus faible ( 0,3 I 5 contre 0,700$)$, après 500 générations on s'attend à trouver encore deux fois plus d'hétérozygotes (KIMURA et CROw, I963, tableau I. p. 403).

Pour ces raisons nous avons étudié spécialement l'évolution de la consangiunité sur une courte période (ro générations) en tenant compte explicitement des relations de parenté existant entre les individus choisis pour constituer la souche témoin, et en envisageant différents schémas possibles caractérisés par un même rapport du nombre de femelles à celui des mâles. Nous avons pris pour référence le schéma des accouplements au hasard, pour lequel nous précisons la définition de la taille efficace de consanguinité. 


\section{I. - Méthodes}

Pour étudier le schéma d'accouplements au hasard nous utilisons la méthode usuelle de Malecot (I948) pour déterminer l'espérance des cœfficients de parenté et de consanguinité moyens à chaque génération. Pour être tout à fait général, il apparaît nécessaire de considérer plusieurs cœfficients de parenté; cela conduit à une définition précise d'une taille effective de consanguinité. Nous avons utilisé les notations de KarLIN (I968).

Pour les autres schémas nous avons eu recours aux cœfficients de consanguinité individuels (CHEVALET, I97I) après avoir construit explicitement le pedigrée correspondant, ou bien un pedigrée possible quand les règles d'accouplement laissent intervenir une part plus ou moins grande de hasard.

\section{I.I. - Calcul en espérance dans le cas des accouplements au hasard}

Dans une population où les accouplements sont faits au hasard, et où les mâles et les femelles sont conservés selon des règles identiques l'équation d'évolution des cœfficients de parenté $\phi$ et de consanguinité $\mathrm{F}$ est donné par :

$$
\begin{aligned}
\phi_{n} & =\frac{\mathrm{I}}{4}\left(p_{m}+p_{f}\right) \frac{\mathrm{I}+\mathrm{F}_{n-1}}{2}+\left[\mathrm{I}-\frac{\mathrm{I}}{4}\left(p_{m}+p_{f}\right)\right] \phi_{n-1} \\
\mathrm{~F}_{n} & =\phi_{n-1}
\end{aligned}
$$

où $p_{m}$ désigne la probabilité que deux individus d'une génération aient un père commun, et $p_{f}$ une mère commune (MAlecot, I948; KARLIN, I968).

Cependant, ces probabilités $p_{m}$ et $p_{f}$ peuvent dépendre des sexes des deux individus considérés. Il est alors nécessaire de définir, à chaque génération, trois cœefficients de parenté :

$$
\begin{aligned}
& \phi_{1}=\text { cœfficient de parenté entre deux mâles } \\
& \phi^{2}=\text { cœfficient de parenté entre deux femelles } \\
& \phi^{3}=\text { cœfficient de parenté entre un mâle et une femelle. }
\end{aligned}
$$

A ces trois cofficients de parenté correspondent trois couples de probabilité $\left(p_{m}^{i}, p_{f}^{i}, i=\mathrm{I}, 2,3\right)$ définissant les probabilités que les individus aient un père et une mère en commun.

De plus, au moins pour la génération des fondateurs il est nécessaire de définir deux cœfficients de consanguinité :

$\mathrm{F}^{\mathbf{1}}=$ cœfficient de consanguinité moyen des mâles

$\mathrm{F}^{2}=$ cœfficient de consanguinité moyen des femelles

On peut alors écrire le système d'équations suivantes :

$$
\begin{aligned}
& \mathrm{F}_{n}^{1}=\phi_{n^{-1}}^{3} \\
& \mathrm{~F}_{n}^{2}=\phi_{n^{-1}}^{3}
\end{aligned}
$$




$$
\left.\begin{array}{rl}
\phi_{n}^{1} & =\frac{\mathrm{I}}{4} p_{m}^{i}\left[\frac{\mathrm{I}+\mathrm{F}_{n^{-1}}^{1}}{2}\right]+\left(\mathrm{I}-p_{m}^{i}\right) \phi_{n^{-1}}^{1} \\
& +\frac{\mathrm{I}}{4} p_{f}^{i}\left[\frac{\mathrm{I}+\mathrm{F}_{n^{-1}}^{2}}{2}\right]+\left(\mathrm{I}-p_{f}^{i}\right) \phi_{n^{-1}}^{2} \\
& +\frac{\mathrm{I}}{2} \phi_{n^{-1}}^{3}
\end{array}\right] i=\mathrm{I}, 2,3
$$

On observe que, même si chez les fondateurs on a $\mathrm{F}_{\mathbf{0}}^{\mathbf{1}} \neq \mathrm{F}_{\mathbf{0}}^{\mathbf{2}}$, l'égalité entre les cœefficients $\mathrm{F}^{\mathbf{1}}$ et $\mathrm{F}^{\mathbf{2}}$ est réalisée dès la première génération et le système peut être réduit à quatre équations. Le système prend une forme plus simple en posant, avec les notations de KARLIN (I968) :

$$
\begin{aligned}
& \mathrm{H}_{n}=\mathrm{I}-\mathrm{F}_{n}^{\mathbf{1}}=\mathrm{I}-\mathrm{F}_{n}^{2}(\text { pour } n \geqslant \mathrm{I}) \\
& \mathrm{G}_{n}^{\mathbf{1}}=\mathrm{I}-\phi_{n}^{\mathbf{1}} \\
& \mathrm{G}_{n}^{\mathbf{2}}=\mathrm{I}-\phi_{n}^{\mathbf{2}} \\
& \mathrm{G}_{n}^{\mathbf{3}}=\mathrm{I}-\phi_{n}^{\mathbf{3}}
\end{aligned}
$$

Sous forme matricielle, le système s'écrit pour $n \geqslant 2$;

(I)

$$
\left[\begin{array}{l}
\mathrm{H}_{n} \\
\mathrm{G}_{n}^{1} \\
\mathrm{G}_{n}^{2} \\
\mathrm{G}_{n}^{3}
\end{array}\right]=\left[\begin{array}{cccc}
\mathrm{o} & \mathrm{o} & \mathrm{o} & \mathrm{I} \\
\mathrm{I} / 8\left(p_{m}^{1}+p_{f}^{1}\right) & \mathrm{I} / 4\left(\mathrm{I}-p_{m}^{1}\right) & \mathrm{I} / 4\left(\mathrm{I}-p_{f}^{1}\right) & \mathrm{I} / 2 \\
\mathrm{I} / 8\left(p_{m}^{2}+p_{f}^{2}\right) & \mathrm{I} / 4\left(\mathrm{I}-p_{m}^{2}\right) & \mathrm{I} / 4\left(\mathrm{I}-p_{f}^{2}\right) & \mathrm{I} / 2 \\
\mathrm{I} / 8\left(p_{m}^{3}+p_{f}^{3}\right) & \mathrm{I} / 4\left(\mathrm{I}-p_{m}^{3}\right) & \mathrm{I} / 4\left(3-p_{f}^{3}\right) & \mathrm{I} / 2
\end{array}\right]\left[\begin{array}{c}
\mathrm{H}_{n-1} \\
\mathrm{G}_{n-1}^{1} \\
\mathrm{G}_{n-1}^{2} \\
\mathrm{G}_{n-1}^{3}
\end{array}\right]
$$

La taille efficace de consanguinité, $\mathrm{N}_{g}$, caractérise dans une population d'effectif constant, la vitesse asymptotique de disparition des hétérozygotes, selon la formule suivante :

(2) $\quad \lim _{n \rightarrow \infty} \frac{\mathrm{I}-\mathrm{F}_{n}}{\mathrm{I}-\mathrm{F}_{n-1}}=\mathrm{I}-\frac{\mathrm{I}}{2 \mathrm{~N}_{g}}$

où $n$ désigne le nombre de générations. Cette quantité $\mathrm{N}_{g}$ est donc associée à la plus grande valeur propre, $\lambda_{m}$, de la matrice définie dans l'équation (I) par :

$$
\mathrm{I}-\frac{\mathrm{I}}{2 \mathrm{~N}_{g}}=\lambda_{m}
$$

On remarque que dans ce cas général où les mâles et les femelles ne sont pas obtenus selon les mêmes règles, la taille efficace $\mathrm{N}_{g}$ ne peut pas être définie simplement par l'inverse de la probabilité $P$ que deux gamètes, pris chez deux individus d'une même génération, proviennent d'un même parent de la génération précédente (définition de Crow et KImURA, I970). En effet cette probabilité $P$ ne peut pas en général être rattachée à la valeur propre $\lambda_{m}$. Au contraire on démontre qu'une expression approchée de $\lambda_{m}$, valable si l'on admet que $\mathbf{I} / \mathrm{N}_{m}^{2}$ et $\mathbf{I} / \mathbf{N}_{f}^{2}$ sont négligeables devant $\mathrm{I}$, fournit pour $\mathrm{N}_{g}$ les expressions données par LATTER (I959), Hill (I972) ou Ollivier (I973). 


\section{I.2. - Calcul explicite des cofficients de consanguinité individuels}

Les systèmes d'accouplements confrontés au schéma d'accouplement au hasard font appel à la constitution de familles entre lesquelles sont réalisés les accouplements. Afin de combiner les avantages théoriques des accouplements circulaires entre sous populations (KIMURA et CROW, I963) et ceux du système de WRIGHT (I92I) nous avons envisagé une extension à des sous populations du système circulaire du troisième degré proposé par CocKERHAM (I970). Nous avons aussi considéré trois variantes d'un système de rotation usuel dans les souches sélectionnées de volaille ou de lapins.

Ces schémas laissent une place plus ou moins grande et parfois nulle, au hasard. Dans tous les cas nous avons réalisé le calcul des cœfficients de consanguinité pour une seule généalogie possible. La représentativité des cœfficients moyens de consanguinité ainsi calculés dépend donc étroitement de la variabilité possible des cœfficients individuels. Cette variabilité, selon WEIR et COCKERHAM (I969), est toujours très faible, elle devient pratiquement nulle après quelques générations, et elle est d'autant plus faible que l'on a évité les accouplements entre individus apparentés. Ces remarques nous permettent de considérer que les cœfficients moyens de consanguinité observés pour une généalogie possible sont bien représentatifs, sauf sans doute pour les toutes premières générations.

Le calcul explicite des cœfficients de consanguinité individuels permet d'observer dans chaque cas étudié leur variabilité dans la souche. Dans les cas où le hasard intervient dans la constitution du pedigree, la variabilité observée ne saurait caractériser le schéma d'accouplement, elle ne représente qu'une possibilité parmi d'autres.

\section{II. - Application des méthodes à un cas particulier}

\section{II.I. - Matériel animal}

En I972, un troupeau de 60 femelles et 12 mâles Néo-Zélandais blancs, fermé depuis r969, a été choisi comme une des souches de base d'une expérience de sélection sur le nombre de lapereaux sevrés par portée, et comme base de la population témoin. En I973, ce troupeau fut porté à I Io reproductrices. Dans ce lot a été tiré un échantillon de 44 femelles accouplées à II mâles différents, à raison de 4 femelles par mâles, 44 filles et II fils de ses reproductrices ont constitué en I974 les animaux de tête de la "souche témoin ".

Les II mâles sont fixés par les origines existantes; puisque un mâle donne un fils, et une femelle une fille, la taille efficace $\mathrm{N}_{g}$ d'après Gowe et al (r959) s'écrit :

$$
\frac{\mathrm{I}}{\mathrm{N}_{g}}=\frac{3}{\mathrm{I} 6 \mathrm{~N} m}+\frac{\mathrm{r}}{\mathrm{I} 6 \mathrm{~N} f}
$$

Si un nombre constant (soit $x$ ) de femelles est accouplé à chaque mâle, $\mathrm{N}_{g}=\frac{\mathrm{r} 76 x}{3 x+\mathrm{I}}$. La valeur maximum possible, obtenue pour $x$ très grand est de 58,67 ; pour les valeurs $x=\mathrm{I}, 2,3,4,5,6$, les valeurs de $\mathrm{N}_{g}$ sont de : 44; 50,3; 52,$8 ; 54,2 ; 55 ; 55,6$. Au-delà de $x=4$, le gain sur $\mathrm{N}_{g}$ apporté par l'addition d'une femelle supplémentaire par mâle devient faible. La population de contrôle fut donc 
constituée d'une cinquantaine d'individus compte tenu aussi de la place disponible pour ce troupeau lors de sa constitution.

Les cœfficients moyens de parenté entre mâles, entre femelles, et entre mâles et femelles sont respectivement :

$$
\phi_{0}^{1}=0,06767 \quad \phi_{0}^{2}=0,06623 \quad \phi_{0}^{3}=0,07045
$$

Dans cette même population initiale, les cœfficients de consanguinité moyens chez les mâles et chez les femelles sont :

$$
\mathrm{F}_{0}^{\mathbf{l}}=0,04490 \quad \mathrm{~F}_{0}^{2}=0,05422
$$

\section{II.2. - Caractérisation des schémas d'accouplements comparés}

Deux types sont mis en comparaison :

- Un schéma où les accouplements sont aléatoires.

- Des schémas à rotation systématique (deux sont étudiés ici).

L'un est une combinaison du schéma circulaire entre sous population de Kimura et Crow (I963) avec le schéma général de Cockerham (I970).

L'autre, qui est le schéma effectivement utilisé dans notre population témoin, adopte un principe des rotations entre familles, analogue à celui réalisé dans les populations sélectionnées et permet donc d'y utıliser les mêmes méthodes statistiques.

\section{Accouplement au hasard.}

Les règles de renouvellement particulières adoptées dans la population permettent d'expliciter les valeurs des probabilités $p_{m}^{i}$ et $p_{f}^{i}$.

$$
\begin{aligned}
& p_{m}^{1}=p_{f}^{1}=0 \\
& p_{m}^{2}=\frac{x-\mathrm{I}}{\mathrm{N}_{m} x-\mathrm{I}}=\frac{3}{43} ; \quad p_{f}^{2}=0 \\
& p_{m}^{3}=\frac{\mathrm{I}}{\mathrm{N}_{m}}=\frac{\mathrm{I}}{\mathrm{II}} ; \quad p_{f}^{3}=\frac{\mathrm{I}}{\mathrm{N}_{m} x}=\frac{\mathrm{I}}{44}
\end{aligned}
$$

L'introduction de ces valeurs dans les équations (I) et la prise en compte des valeurs initiales des cœfficients de parenté et de consanguinité permet le calcul en espérance de la consanguinité. A ce cas (désigné comme cas no I dans la suite), nous avons joint les résultats d'un calcul explicite des cœfficients de consanguinité individuels sur une généalogie possible ( $\operatorname{cas} n^{\circ} 2$ ).

\section{Accouplements circulaires du troisième degré entre familles.}

Ce schéma (cas $\mathrm{n}^{\mathbf{0}} 3$ ) est construit selon la règle suivante, élaborée d'après les études de Kimura et Crow (I963) et de Cockerham (I970) : la population est fractionnée à chaque génération $n$ en $\mathrm{N}_{m}$ groupes $\mathrm{G}_{i}^{n}$ dont chacun comprend un mâle et $x$ femelles; à la génération $(n)$ les $x$ femelles du groupe $\mathrm{G}_{i}^{n}$ sont accouplées au mâle du groupe $\mathrm{G}_{i}^{n}$, (où $i^{\prime}$ est de la forme $i^{\prime}=i+2^{n}$, modulo $\mathrm{N}_{m}$ ); Une fille de chacune des femelles du groupe $\mathrm{G}_{i}^{n}$ est affectée au groupe $\mathrm{G}_{i}^{n+1}$ de la génération 
suivante, le mâle du groupe $\mathrm{G}_{i}^{n+1}$ est tiré au hasard dans la descendance mâle des femelles du groupe $\mathrm{G}_{i}^{n}$.

Par conséquent la généalogie sur laquelle ont été calculés les cœfficients de consanguinité individuels représente une généalogie possible puisque le choix de la mère de chaque mâle reste aléatoire.

\section{Accouplements pratiqués dans la souche témoin.}

On suppose l'existence de groupes de reproduction $(G)$ constitués de un mâle et de 4 femelles. Le plan de constitution de la génération $n+\mathrm{I}$ à partir des II groupes $\left(\mathrm{G}_{i}^{n}\right)$ de la génération $n$ (fig. $\mathrm{I}$ ) suit la règle suivante : seule.

a) Tout mâle laisse un fils et un seul; toute femelle laisse une fille et une

b) Le mâle du groupe $\mathrm{G}_{i}^{n+1}$ est le fils du groupe $\mathrm{G}_{i}^{n}$. Sa mère est une des 4 femelles du groupe $\mathrm{G}_{i}^{n}$. Dans le plan d'accouplement ainsi présenté, seul le choix de la mère de chaque mâle n'est pas précisé. La mère du mâle du groupe $\mathrm{G}_{i}^{n}$ est l'une des femelles du groupe $\mathrm{G}_{i}^{n-1}$. On peut alors caractériser celle-ci par son numéro d'ordre $(j)$ dans ce groupe. Trois cas ont été envisagés :

Cas $n^{0} 4$ : dans chaque groupe, l'indice $j$ de la mère du mâle est tiré au hasard parmi les quatre valeurs possibles $j=\mathrm{r}, 2,3$ ou 4 ; c'est pratiquement ce qui se passe à chaque génération dans la souche témoin lapin.

Cas $n^{0} 5$ : l'indice $j$ de la mère du mâle du groupe $\mathrm{G}_{i}^{n}$ est défini systématiquement par la formule $: j-\mathrm{I}=i+n$ (modulo 4 );

Cas $n^{\circ}$ 6: l'indice $j$ prend une valeur constante pour tous les groupes et toutes les générations (nous avons fixé $j=3$ ).

Les cas 5 et 6 définissent exactement la généalogie; pour le cas 4 une généalogie possible a été construite en réalisant des tirages aléatoires de l'indice $j$ : les cas 5 et 6 représentent deux possibilités extrêmes rendues possibles par la règle du cas no 4 .

c) Les 4 femelles du groupe $\mathrm{G}_{i}^{n+1}$ sont issues de 4 groupes différents, $\mathrm{G}_{i-1}^{n}$, $\mathrm{G}_{i-2}^{n}, \mathrm{G}_{i^{-3}}^{n}, \mathrm{G}_{i_{-4}}^{n}$. Cela revient à dire que les 4 femelles du groupe $\mathrm{G}_{i}^{n}$ donnent 4 filles; la première va dans le groupe $G_{i+1}^{n+1}$, la seconde dans le groupe $G_{i+2}^{n+1}$; la troisième dans le groupe $\mathrm{G}_{i+3}^{n+1}$ et la quatrième dans le groupe $\mathrm{G}_{i+4}^{n+1}$.

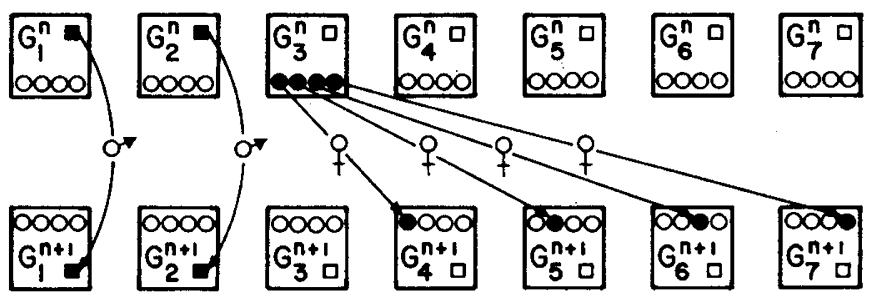

FIG. I. - Constitution des groupes de reproduction de la génération $n+\mathbf{I}$ à partiv des individus issus des groupes de reproduction de la génération $n$.

FIG. I. - Distribution in $(n+\mathrm{I})$-th generation of offspring from the $n-t h$ generation for the control line. 
d) Les femelles ont, dans un groupe donné, un numéro d'ordre que conserve leur fille dans le groupe où elle se situe à la génération suivante.

Si l'on représente dans la figure I l'ensemble de la constitution de la génération $n+\mathrm{I}$, celui-ci devient d'une lecture très complexe et peut donc sembler peu aisé à réaliser. Lors de la mise en place d'une génération de la population de contrôle, on peut représenter plus simplement les accouplements, en rangeant les individus mâles et femelles en familles de demi frères ou sœurs de père (fig. 2).
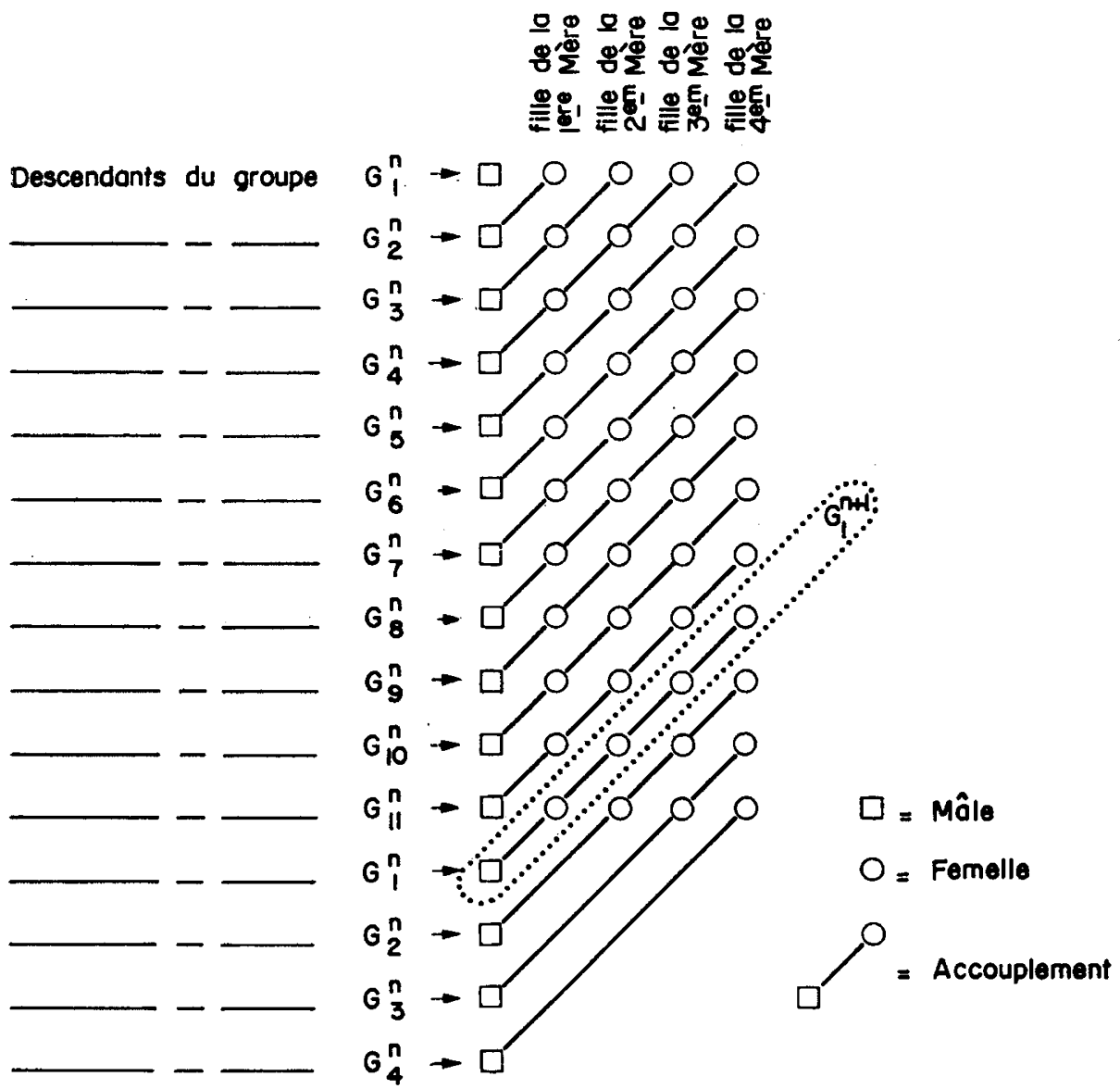

FIG. 2. - Réalisation pratique des groupes de reproduction une fois les animaux rangés en familles de pères.

Fig. 2. - Actual vealization of breeding groups between offspring.

Males are represented by squares, females by circles; each row is made of halfsibs; matings are made between one male and four females of the same diagonal.

\section{III. - Résultats}

L'évolution du cœfficient de consanguinité moyen est présenté à la figure 3 pour les dix premières générations et pour les six cas étudiés. Hormis les deux premières générations, les différents schémas présentent des évolutions bien diffé- 
renciées. Les fluctuations initiales peuvent être dues à deux facteurs : d'une part la constitution initiale des groupes d'individus a été réalisée de façon à éviter des accouplements consanguins à la première génération, compte tenu des parentés initiales connues et du schéma (cas no ${ }^{\circ}$ ) adopté; d'autre part, pour les cas 2,3 et 4 , la généalogie réalisée ne représente qu'une possibilité, le cœfficient moyen observé ne peut être assimilé à une espérance. Au-delà de la troisième génération, au contraire, il est légitime de considérer les moyennes obtenues (dans les cas $2,3,4$ ) comme de bonnes estimations des espérances, compte tenu des remarques de WEIR et Cockerham (I969). Les différents cas étudiés se distinguent par les évolutions des valeurs moyennes de la consanguinité, mais aussi par la variabilité des cœfficients de consanguinité individuels autour de ces moyennes (tableau I, figure 3).

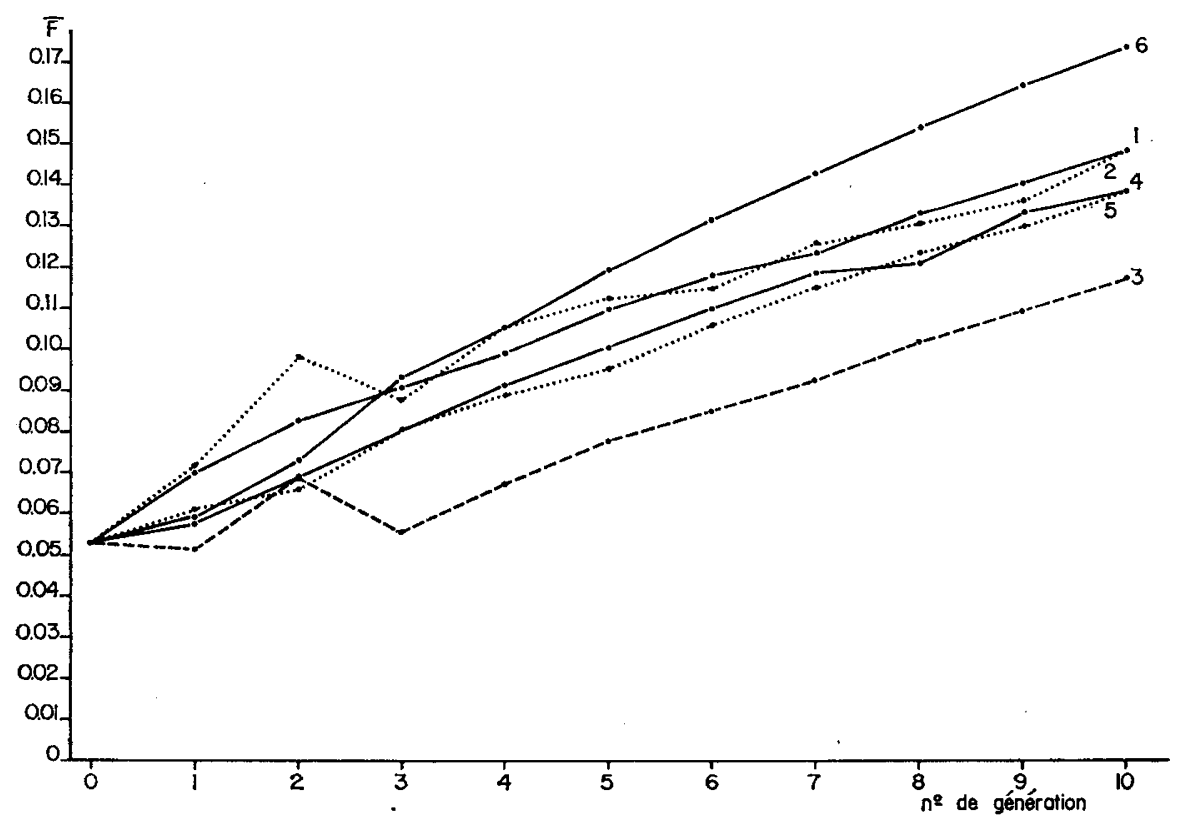

Frg. 3. - Evolution probable de la consanguinité moyenne dans la souche témoin de lapins de l'élevage expérimental d'Auzeville.

FIG. 3. - Expected evolution of mean inbreeding coefficients with time, for the six compared designs.

Numbering as in Table I. Abcsissa : generation number; ordinates : values of inbreeding $\mathrm{F}$ coefficients).

\section{III.x. - Évolution des moyennes}

Entre la troisième et la dixième génération, l'accroissement moyen du cœffcient de consanguinité par génération est comparable pour les cinq premiers cas étudiés $(0,8 \mathrm{I}$ à $0,87 \mathrm{p}$. Ioo contre $\mathrm{I}, \mathrm{I} 7 \mathrm{p}$. Ioo pour le cas 6$)$ et correspond à peu près à l'accroissement moyen calculable d'après la taille efficace $\mathrm{N}_{g}=54,2$ calculée pour les cas $n^{0}$ I et 2 . Il est remarquable d'observer le maintien, pendant ce laps de temps, des différences réalisées à la troisième génération :

+ I p. Ioo pour le hasard (cas $\mathrm{n}^{\circ} \mathrm{I}$ et 2 ) et -2 p. Ioo pour le schéma minimisant la valeur moyenne de $\mathrm{F}$ ( $\operatorname{cas} n^{\circ} 3$ ), par rapport aux cas no 4 et 5 de la souche témoin. 
TABLEAU I

Distribution du coefficient de consanguinité individuel selon le cas (à la génération 3 et Io)

Minimum and maximum values per sex, mean values, and standard deviations of inbreeding coefficients in the third and tenth generations. Various cases are numbered as follows :

I. Theoretical expected value under random mating.

2. Observed values in one simulated run of random mating.

3. Third degree subpopulation circular system.

4, 5 and 6 : various designs for the control line, according to rules shown in figures $I$ and 2 .

\begin{tabular}{|c|c|c|c|c|c|c|c|c|}
\hline & \multirow{2}{*}{ No du cas } & \multicolumn{2}{|c|}{$\mathrm{F}$ minimum } & \multicolumn{2}{|c|}{$\mathrm{F}$ maximum } & \multirow{2}{*}{$E(F)$} & \multirow{2}{*}{$\overline{\mathrm{F}}$} & \multirow{2}{*}{$\sigma_{\mathbf{F}}$} \\
\hline & & & & & & & & \\
\hline \multirow{6}{*}{ 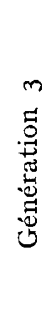 } & I & & & & & 0,090 & & \\
\hline & 2 & 0,039 & 0,039 & 0,184 & 0,256 & & 0,088 & 0,047 \\
\hline & 3 & 0,049 & 0,042 & 0,074 & 0,074 & & 0,056 & 0,007 \\
\hline & 4 & 0,048 & 0,038 & 0,127 & 0,128 & & 0,080 & 0,022 \\
\hline & 5 & 0,054 & 0,049 & 0,088 & $0, \mathrm{I} 3 \mathrm{I}$ & 0,080 & & 0,020 \\
\hline & 6 & 0,124 & 0,037 & $0, I_{4} \mathrm{I}$ & $0,1_{4} r$ & 0,092 & & 0,034 \\
\hline \multirow{6}{*}{ 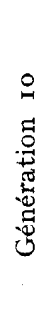 } & $\mathrm{r}$ & & & & & 0,149 & & \\
\hline & 2 & $0, \mathrm{II}_{4}$ & 0,112 & 0,237 & 0,268 & & $0, \mathbf{1} 48$ & 0,040 \\
\hline & 3 & O,II6 & 0,116 & 0,120 & 0,120 & & 0,117 & 0,001 \\
\hline & 4 & $0, \mathrm{II}_{5}$ & O,III & $0, \mathrm{I} 73$ & 0,205 & & 0,139 & 0,020 \\
\hline & 5 & o, I I 5 & O, I I 5 & $\mathrm{o}, \mathrm{I} 42$ & 0,192 & 0,139 & & 0,020 \\
\hline & 6 & $0,27 \mathrm{I}$ & 0,097 & 0,279 & 0,279 & 0,174 & & 0,083 \\
\hline
\end{tabular}

Le cas $n^{\circ} 4$ où l'on constitue une généalogie possible parmi d'autres, ne se différencie pas du cas $\mathrm{n}^{0} 5$ où la rotation des mères de pères est systématique. Cela peut cependant être un cas fortuit; une autre issue, peu probable il est vrai, est le cas no 6 qui se distingue par une augmentation nettement plus rapide de la consanguinité.

\section{III.2. - Variabilité de la consanguinité individuelle}

Sauf pour les cas 5 et 6 , les valeurs indiquées ne sont qu'indicatives puisque tirées d'une généalogie particulière. La tendance observée dans le cas $\mathrm{n}^{0} 3$ vers une homogénéisation des cœefficients de consanguinité individuels peut cependant être considérée comme significative, elle n'est qu'une illustration d'un résultat connu sur les systèmes qui évitent les accouplements entre apparentés jusqu'à un degré fixé (Weir et Cockerham, ig69; Cockerham, I97o). 
Les distributions observées à la dixième génération sont indiquées au tableau I.

Les valeurs minimales sont toujours à peu près les mêmes (o, Io à 0,12$)$. Une plus grande variabilité est à noter sur les valeurs maximales (o, I5 à 0,27$)$. Le schéma $n^{0} 3$ uniformise tous les cœfficients individuels, les valeurs maximales et minimales sont identiques. Le schéma adopté pour notre souche témoin (cas $\mathrm{n}^{0} 4$ ) et le cas particulier $n^{\circ} 5$ semble apporter une plus grande homogénéité que le schéma au hasard (écart-type diminué de moitié); en outre la distribution des cœfficients de consanguinité individuels montre que ceux-ci se répartissent à peu près unjformément dans tous l'intervalle (minimum-maximum).

Le schéma $n^{\circ} 6$ donne une structure intéressante : en fait on y trouve deux sous populations distinctes et homogènes, l'une contenant $60 \mathrm{p}$. Ioo des animaux (ce ne sont que des femelles) qui ont la consanguinité la plus faible de tous les schémas $\left(9,7 \mathrm{p}\right.$. IOO) et l'autre avec $4^{\circ} \mathrm{p}$. Ioo des animaux (tous les mâles et quelques femelles) avec la plus forte (28 p. IOO) (fig. 4).

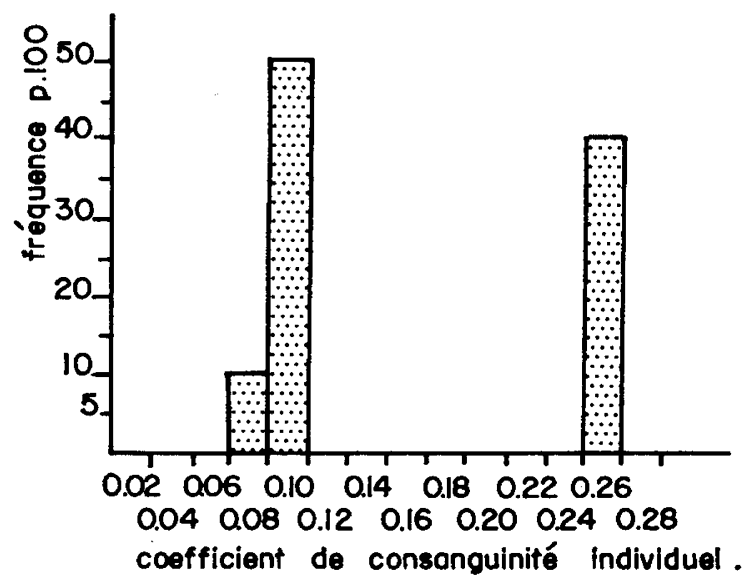

FIG. 4. - Distribution des fréquences du coeffcient de consanguinité individuel dans le cas $n^{\circ} 6$ à la I $\mathrm{o}^{\mathrm{e}}$ génération.

FIG. 4. - Histogram of individual inbreeding coefficient values in tenth generation, case number 6.

\section{Discussion - Conclusion}

Dans l'étude précédente, et pour l'intervalle de ro générations considéré, tous les schémas comparés (sauf le sixième) présentent une même vitesse d'évolution et se distinguent par les niveaux moyens de consanguinité atteints à la troisième génération. De ce point de vue, le schéma du témoin (cas 4 ou 5) est intermédiaire entre le système d'accouplements au hasard (cas I et 2) et le système qui minimise la consanguinité (cas $n^{\circ} 3$ ). Le schéma $n^{\circ} 5$ assure aussi une moindre variabilité des cœefficients de consanguinité individuels que le système d'accouplement au hasard, cela peut être un avantage si une consanguinité individuelle forte est défavorable à la survie ou à la reproduction d'un individu. Avoir moins d'individus très consanguins représente un moindre risque de disparition de la population.

La dérive génétique peut être caractérisée par l'espérance de la fluctuation de la fréquence $q$ d'un allèle à partir de sa valeur initiale $q_{0}$. Dans une population idéale panmictique où le nombre de descendants par parent suit une loi de Poisson, 
cela peut s'exprimer pour des effectifs $\mathrm{N}_{m}$ et $\mathrm{N}_{f}$ constants en fonction de la consanguinité moyenne :

$$
\operatorname{var}\left(q_{n}-q_{0}\right)=q_{0}\left(\mathrm{I}-q_{0}\right) \mathrm{F}_{n}
$$

à une génération donnée (FALCONER, I960). Plus généralement, si l'effectif est constant les tailles efficaces de "consanguinité " et de "variance " sont identiques (Crow et Kimura, I970; Wright, I969), il revient donc alors au même de minimiser la dérive ou la consanguinité. Si l'on a des accouplements au hasard mais une structure particulière, il faut remplacer $\mathrm{F}_{n}$ par une combinaison linéaire de $F_{n}$ et des cofficients de parenté $\phi_{n}^{1}, \phi_{n}^{2}, \phi_{n}^{3}$ à la génération $n$; mais la dérive doit rester du même ordre de grandeur. Cela devient plus compliqué sı l'on prend une structure spéciale comme celle du schéma $\mathrm{n}^{\circ} 6$.

La comparaison entre schémas et l'intérêt de chacun suppose que l'on puisse les réaliser exactement selon les prévisions. Or cela n'est pas certain dans tous les cas : une mère peut ne pas laisser de fille viable, une famille de père peut disparaître. Les conséquences de tels événements peuvent être appréhendées sur le long terme en étudiant la valeur de $\mathrm{N}_{g}$ pour les accouplements aléatoires : si l'on admet par exemple que l'on obtient la distribution suivante :

II fils et 44 filles avec la probabilité I $/ 5$.

II fils et 33 filles avec la probabilité I $/ 5$.

Io fils et 44 filles avec la probabilité $I / 5$.

Io fils et 33 filles avec la probabilité $\mathbf{r} / 5$.

9 fils et 38 filles avec la probabilité I $/ 5$.

alors on peut définır un $\mathrm{N}_{g}$ moyen par génération en prenant la moyenne harmonique (Malecot, I948) qui donne $\mathrm{N}_{\boldsymbol{g}}^{\prime}=49,65$ au lieu de 54,I5. Cela ne semble pas avoir de conséquences très grandes si l'on considère l'évolution moyenne sur plusieurs générations. Mais à court terme il n'est pas exclu que des modifications aléatoires apportées au schéma théorique aient des conséquences non négligeables. La réalisation pratique des générations successives de la population témoin permettront d'évaluer la fréquence de tels événements et d'en mesurer les conséquences puisqu'il sera possible de comparer l'évolution réalisée de la consanguinité à celle attendue dans l'hypothèse de réalisation stricte du plan d'accouplements prévu.

$$
\text { Reçu pour publication en février } 1977 .
$$

\section{Summary \\ Design of a control line of rabbits \\ Expected evolution of individual inbreeding coefficients according to various mating schemes.}

Several designs are compared for a control line of rabbits, with respect to the evolution of inbreeding. Two types of circular matings between eleven families are compared to the random mating scheme with the same renewal rules. From the theoretical point of view, a precise definition of an inbreeding effective size is given for those cases where the distribution of offspring number per parent depends upon offspring's sex : in such a situation, inbreeding effective size should not be defined as the reciprocal of the probability that two gametes, drawn out two zygotes of a generation, derive from one common parent of the preceding generation. For practical purpose, emphasis is put on the behaviour during first generations and on the influence of initial relationships between founders, account of which cannot be taken through the only effec- 
tive size of the population. Schemes are compared during ten generations from the generating of pedigrees and the computing of individual inbreeding coefficients. Comparisons could therefore be made with respect to both mean values and fluctuations of inbreeding coefficients. One scheme was found to be the better one, it combines the properties of the circular subpopulation mating, due to KrMURA and Crow (1963), and of the third degree circular system defined by CoCKERHAM (1970). Starting with the same related ancestors, it allows a permanent reduction of the mean inbreeding coefficients, of $3 \mathrm{p}$. Ioo when compared to random mating; after a few generations it makes all coefficients equal, avoiding any random appearance of highly inbred individuals.

\section{Références bibliographiques}

Bulletin Technique du Département de Génétique Animale, 1975. Mesure du progrès génétique réalisé par sélection sur les animaux de ferme, $\mathrm{n}^{\circ} \mathrm{I} 9$.

Chevalet C., 1971. Calcul automatique des coefficients d'identité. Ann. Génét. Sél. Anim., 3, 449-462.

Cockerham C. C., 1970. Avoidance and rate of inbreeding. Kojima K. (ed.), Mathematicat topics in population genetics, Springer Verlag, Berlin.

Crow J. F., Kimura M., I970. Introduction to population genetics theory. Harper and Row, New-York.

FALCONER D. S., 1960. Introduction to quantitative genetics. Oliver an Boyd. London.

Gowe R. S., Robertson A., Latter B. D. M., I959. Environnement and poultry breeding. problems, 5. The design of poultry control strains. Poultr. Sci., 38, 462-47r.

HILL W. G., 1972. Estimation of genetic change. I. General theory and design of control populations. Anim. Breeding Abstr., 40, I-I5.

KARLIN S., 1968. Equilibrium behavior of population genetic models with non-random mating. Part II. Pedigrees, homozygosity and stochastic models. J. appl. Prob., 5, 487-566.

KimURA M., Crow J. F., I963. On the maximum avoidance of inbreeding. Genet. Res., Lamb., 4, 399-4I 5 .

LAtTER B. D. H., I959. Genetic sampl ing in a random mating control population of constant size and sex ratio. Aust. J. Biol. Sci., 12, 500-505.

Malecot G., 1948. Les mathématiques de l'hérédité. Masson et Cie. Paris.

Ollivier L., 1973. Le calcul de l'effectif génétique des populations animales. Ann. Génét. Sél. Anim., 5 (3), 363-368.

Weir B. S., Cockerham C. C., I969. - Group inbreeding with two linked loci. Genetics, 63 , 7II-742.

WRIGHT S., I921. Systems of mating. Genetics, 6, III-I 78 .

WRrGHT S., 1969. Evolution and the genetics of populations, vol. 2. The theory of gene frequencies. University of Chicago Press, Chicago; chap. 7, 8, 12. 